\title{
Direct Measurement of the Confining Forces Imposed on a Single Molecule in a Concentrated Solution of Circular Polymers
}

\author{
Rae M. Robertson and Douglas E. Smith* \\ Department of Physics, University of California-San Diego, Mail Code 0379, 9500 Gilman Drive, \\ La Jolla, California 92093
}

Received June 28, 2007; Revised Manuscript Received September 7, 2007

\begin{abstract}
We measure the forces confining the displacement of a single DNA molecule embedded within a concentrated solution of long relaxed circular DNA molecules (115 kbp at $1 \mathrm{mg} / \mathrm{mL}$ ) using optical tweezers. We compare these measurements with our previous measurements of forces imposed by entangled linear DNA molecules of the same length and concentration. A tube-like confining field and three relaxation modes were observed, but the tube radius was $25 \%$ smaller $(\cong 0.6 \mu \mathrm{m})$ and the longest relaxation time $\sim 3$ times shorter $(\cong 11$ s) than with linear molecules, consistent with recent theoretical predictions by Iyer, Lele, and Juvekar. For displacements greater than the tube radius, the confining force imposed by circular polymers was substantially lower and shorter range than that measured with linear polymers.
\end{abstract}

\section{Introduction}

Intermolecular entanglements exert strong effects on the molecular dynamics and bulk fluid properties of concentrated polymer solutions. The reptation model, developed by de Gennes, Doi, Edwards, and others, has been essential in understanding such effects, especially as applied to linear polymers. ${ }^{1-3}$ Circular polymers, such as DNA, present a more puzzling case, however. Their configurations and entanglements are less well understood, and it is not clear to what extent reptation theory applies. The key assumption of reptation theory is that entanglements act on short time scales to confine each molecule in a tube-like region tangent to its contour. While linear polymers can diffuse either head- or tail-first out of their tubes, forming new displaced tubes, circular polymers have no ends by which to escape tube constraints by conventional reptation.

Studies of synthetic circular polymers have, in some cases, found substantial differences in physical properties such as molecular diffusion and fluid rheology, as compared with linear polymers. ${ }^{4-10}$ However, a consensus understanding of the existing data has not been reached. In the case of DNA, we recently measured diffusion coefficients for entangled linear and relaxed circular molecules by tracking the Brownian motion of single molecules with fluorescence microscopy. ${ }^{11} \mathrm{We}$ found that the scaling of the diffusion coefficient with concentration and length was consistent with the reptation model for both topologies, but relaxed circular DNA diffused up to 7 times faster than linear DNA of the same length and concentration (45 $\mathrm{kbp}$ at $1 \mathrm{mg} / \mathrm{mL}$ ). Thus, relaxed circular DNA appears to be less effective than linear DNA at producing restrictive entanglements.

We recently developed a method for directly measuring the intermolecular forces imposed by entangled DNA molecules using optical tweezers. ${ }^{12}$ Our initial studies only examined linear molecules. Here, we extend this method to study entangled circular molecules and report significantly different results than with linear DNA. In our technique, a stretched probe molecule is displaced within an entangled DNA solution while measuring the induced force (Figure 1). In our studies with linear DNA, the force opposing displacement parallel to the probe molecule

\footnotetext{
* Corresponding author. E-mail:des@physics.ucsd.edu.
}

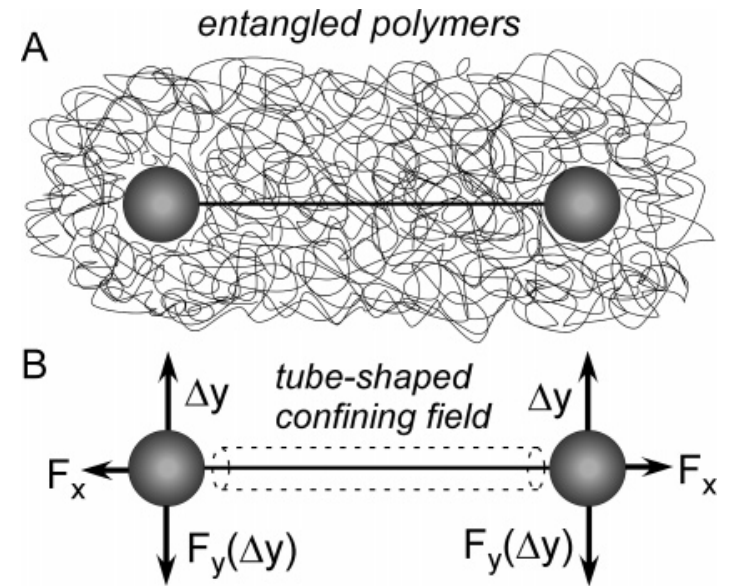

Figure 1. Schematic diagram of the experiment. (A) A single DNA molecule is held stretched between two optically trapped microspheres and suspended in a concentrated solution of circular DNA. (B) Reptation models postulate that collective intermolecular interactions give rise to a tube-shaped confining field (dashed lines).

was found to be negligible, but we measured a significant transverse force. We found that the transverse confining potential per unit length was roughly $1 k T$ at a displacement distance similar to the theoretically predicted tube radius, in accord with predictions of recent molecular dynamics simulations. ${ }^{13}$ We also found that the effective tube radius increased with time, also in agreement with the simulations. ${ }^{13}$ In addition, we measured the force relaxation and observed three relaxation modes. The shortest relaxation time corresponded to the calculated Rouse time $\left(\tau_{\mathrm{R}}\right)$ and the longest to the calculated disengagement time $\left(\tau_{\mathrm{D}}\right)$ in the Doi-Edwards (D-E) model. $^{2}$ An intermediate relaxation time was also observed, consistent with a relaxation mechanism proposed by Mhetar and Archer. ${ }^{14}$

Some theories predict that entangled circles adopt a tree structure resembling a randomly branched polymer, and it has been conjectured that they could diffuse by reptation with protruding finger-shaped loops that continually thrust forward and back, in an amoeba-like fashion. ${ }^{15-18}$ Several simulations of circular polymer melts find evidence for a lower degree of entanglement and higher threshold length for entanglement than for linear molecules. ${ }^{19-23}$ Recently, Iyer et al. formulated a 
detailed dynamical theory for entangled circular polymers using concepts from the pom-pom model, previously used to describe branched polymers within a tube model framework. ${ }^{24,25}$ Within the pom-pom ring (PPR) framework of Iyer et al., an entangled circular polymer adopts a lattice-tree structure (akin to a randomly branched polymer but formed from loops) consisting of a primary/longest length ("trunk") and shorter, randomly positioned loops ("branches"). The longest time scale dynamics of such a structure is modeled as being governed by a reptationtype motion of the trunk (with predicted length $\sim N^{2 / 3}$, where $N$ is the length of polymer) with added friction from the smaller loop branches, which are unentangled and able to relax more quickly. ${ }^{26} \mathrm{We}$ note that these existing theories have all been aimed at describing melts. However, tube models for linear polymer melts have been successfully extended to analyze solutions of entangled polymers using "blob" theory, ${ }^{1,27,28}$ in which each chain is divided into correlation blobs of length $\zeta$ and the solution is modeled as a melt of chains with effective monomer size $\zeta$. It is possible that blob theory may also be applied to circular polymers.

According to $\mathrm{D}-\mathrm{E}$ theory, the confining tube radius is given by $a=\left(24 / 5\left(N_{\mathrm{e}} / N\right) R_{\mathrm{G}}{ }^{2}\right)^{1 / 2}$, where $R_{\mathrm{G}}$ is the radius of gyration and $N_{\mathrm{e}}$ the molecular length per entanglement. ${ }^{2}$ A rigorous theoretical definition of $N_{\mathrm{e}}$, specified in terms of molecular properties, has proven elusive, so $N_{\mathrm{e}}$ is usually an empirically determined quantity inferred from measurements of the bulk plateau modulus $G_{\mathrm{N}}{ }^{(0)}$ of polymer solutions. On the other hand, efforts have recently been made to define and quantify entanglements directly through molecular dynamics simulations. ${ }^{29-33}$ Zhou and Larson have recently used such an approach to directly calculate the tube confining potential by analyzing an ensemble of simulated molecular trajectories, ${ }^{13}$ and our measurements on linear DNA compare favorably with some of their predictions. ${ }^{12}$

According to the PPR model proposed by Iyer et al., the relative size of the tube radius for circular polymers compared with linear polymers depends on $N$ as well as the ratio of the corresponding $N_{\mathrm{e}}$ values for both topologies (i.e., $N_{\mathrm{eC}} / N_{\mathrm{eL}}$, where the $\mathrm{C}$ and $\mathrm{L}$ subscripts refer to the values for linear and circular polymers, respectively). ${ }^{26}$ They derive the expression $a_{\mathrm{C}}{ }^{2} / a_{\mathrm{L}}{ }^{2}$ $=\left(N_{\mathrm{eC}} / N_{\mathrm{eL}}\right) N^{2 v-1}$, where $v \cong 0.3$ is the static scaling exponent predicted for semiflexible polymers such as DNA. ${ }^{21} N_{\mathrm{eC}} / N_{\mathrm{eL}}$ has not been predicted theoretically, so Iyer et al. assume a value of $N_{\mathrm{eC}} / N_{\mathrm{eL}} \cong 5$, deduced from rheological measurements on polystyrene melts. ${ }^{5}$ According to $\mathrm{D}-\mathrm{E}$ theory, for linear DNA the tube radius is predicted to be $a=\left(24 / 5\left(N_{\mathrm{e}} / N\right) R_{\mathrm{G}}{ }^{2}\right)^{1 / 2} \cong 0.5$ $\mu \mathrm{m}$, using measured values of $R_{\mathrm{G}}$ and $G_{\mathrm{N}}{ }^{(0)} \cdot{ }^{12,34-37}$ Thus, for circular DNA the theory of Iyer et al. predicts $a_{\mathrm{C}} \cong 0.7 a_{\mathrm{L}} \cong$ $0.3 \mu \mathrm{m}$. As will be described below, this prediction compares favorably with our measurements.

\section{Materials and Methods}

The details of our experiment are essentially the same as in our previous study, ${ }^{12}$ except that a solution of relaxed circular DNA was probed instead of linear DNA. The probe molecule was a 25.3 kbp DNA $(8.4 \mu \mathrm{m})$ construct prepared and labeled with biotin on one end and digoxygenin (DIG) on the other using PCR as described previously. ${ }^{38}$ The probe was suspended in a $1 \mathrm{mg} / \mathrm{mL}$ solution of $115 \mathrm{kbp}$ DNA molecules and tethered by attaching the biotin end to an optically trapped streptavidin microsphere and the DIG end to an optically trapped anti-DIG coated microsphere, as described previously. ${ }^{38}$ The tethered probe was stretched with a tension of $10 \mathrm{pN}$. The $115 \mathrm{kbp}(38 \mu \mathrm{m})$ molecules were prepared by cloning in E. coli. ${ }^{39}$ These molecules were treated with topoisomerase I to produce torsionally relaxed, non-interlinked circles. ${ }^{34}$ Displacements were made by moving the sample chamber with a piezoelectric nanopositioning stage, and the induced force along the axis of
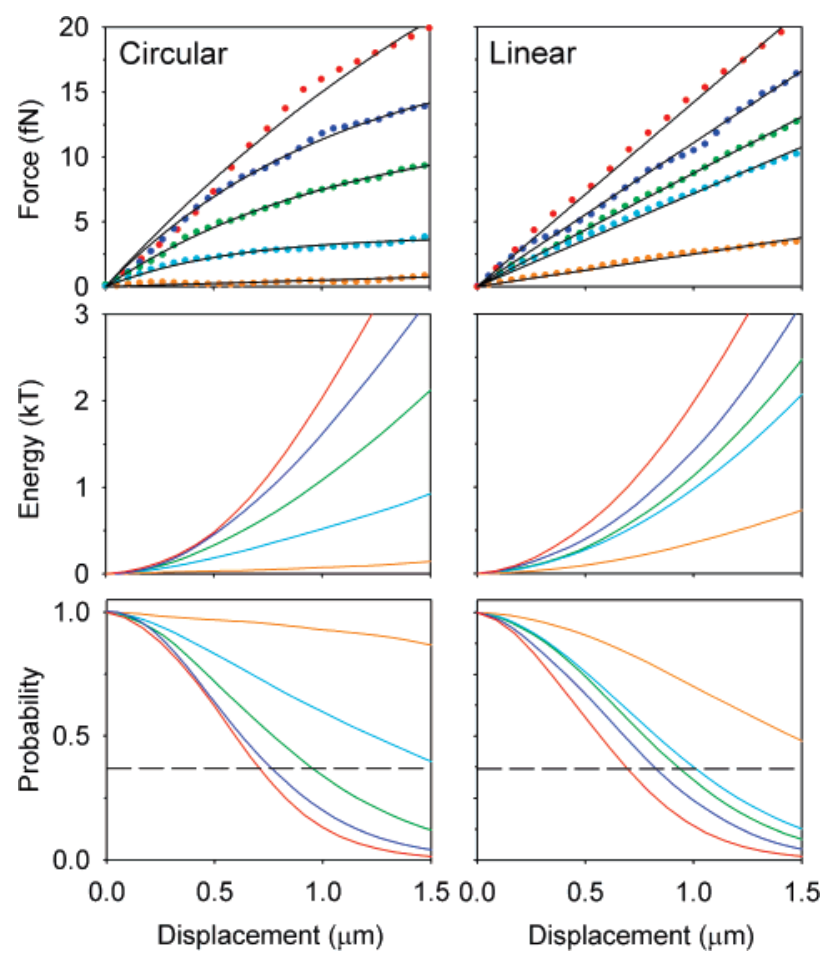

Figure 2. (Top-left) Induced force per unit length $\left(F_{y}\right)$ measured during a small displacement $\Delta y$ at rates of $65 \mu \mathrm{m} / \mathrm{s}$ (red), $25 \mu \mathrm{m} / \mathrm{s}$ (blue), 13 $\mu \mathrm{m} / \mathrm{s}$ (green), $0.52 \mu \mathrm{m} / \mathrm{s}$ (cyan), and $0.10 \mu \mathrm{m} / \mathrm{s}$ (orange); color schemes are the same for the other panels. The lines are fits to the function $F_{y}$ $=\alpha\left(1-\mathrm{e}^{-\beta \Delta y}\right)$. (Top-right) Forces measured with linear DNA of the same length and concentration in our previous study. ${ }^{12}$ The lines are linear fits. (Middle-left) Confining potential per unit length $U(y)$ determined by integration of the force data for circular DNA. (Middleright) Potential measured with linear DNA. (Bottom-left) Relative probability $P(y)=\mathrm{e}^{-U(y) / k T}$ for the chain segments to fluctuate a transverse distance $y$ from the primitive path. Inferred tube radii determined as the displacements where $P(y)=1 / \mathrm{e}(U=1 k T)$, indicated by the positions where the curves cross the dashed line $(0.70,0.76$, 0.95 , and $1.6 \mu \mathrm{m}$ for the $65,25,13$, and $0.52 \mu \mathrm{m} / \mathrm{s}$ rates, respectively). (Bottom-right) Relative probability plotted for linear DNA. The inferred tube radii are $0.65,0.82,0.94,1.0$, and $1.8 \mu \mathrm{m}$ for the $65,25\left(a_{\mathrm{L}} / \tau_{\mathrm{eL}}\right)$, $13,0.52$, and $0.10 \mu \mathrm{m} / \mathrm{s}$ rates, respectively.

displacement was measured by recording the deflection of the trapping laser, as described previously. ${ }^{12,40}$ A laser power of 150 $\mathrm{mW}$ was used in each trap, which causes minimal local heating, from $\sim 22^{\circ} \mathrm{C}$ ambient temperature to $\sim 27^{\circ} \mathrm{C}$. This increase is minor in that it does not cause any structural changes in the DNA (since it is only expected to melt at a much higher temperature of $\sim 90$ ${ }^{\circ} \mathrm{C}$ ), and it changes the thermal energy $k T$ (where $T$ is in kelvin) by only $1.6 \%$. Previously, to obtain a confining force per unit length, independent of the arbitrary probe length, we normalized the total induced force by the number of correlation blobs spanned by the probe molecule $(L / \zeta=8.4 \mu \mathrm{m} / 52 \mathrm{~nm}=162) .{ }^{12} \mathrm{In}$ order to directly compare our findings for circular DNA to those of linear DNA, we chose to normalize the data for circular DNA by the same quantity.

\section{Results and Discussion}

Transverse displacements were imposed at rates of $0.10,0.52$, 13,25 , and $65 \mu \mathrm{m} / \mathrm{s}$, as in our previous study with linear DNA. ${ }^{12}$ The results with circular and linear DNA are compared in Figure 2. For small displacements $(\Delta y<0.4 \mu \mathrm{m})$, the forces were approximately the same for both topological cases and increased linearly with displacement for the higher displacement rates (13-65 $\mu \mathrm{m} / \mathrm{s})$. The induced force was rate-dependent, as discussed previously. ${ }^{12}$ A Hookean response was observed with 


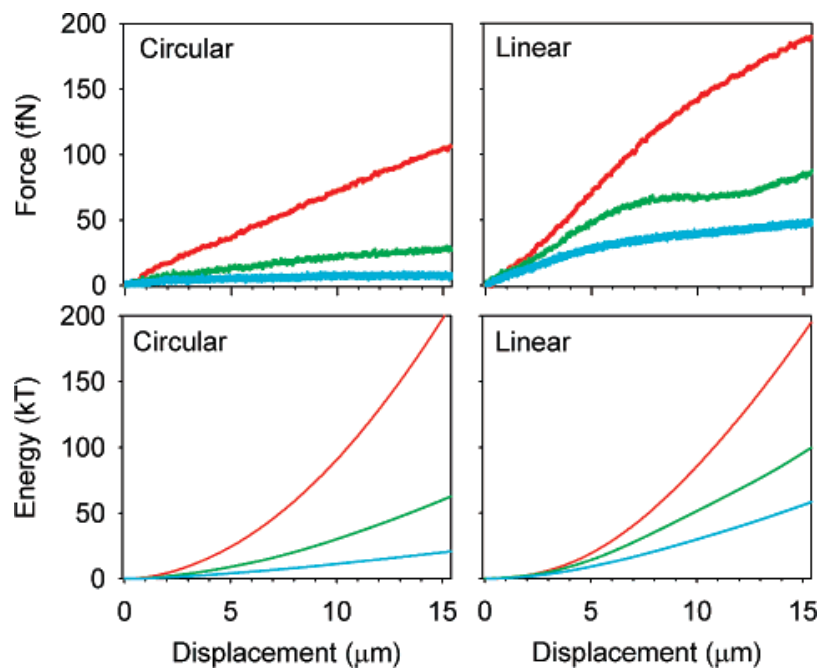

Figure 3. Induced force during a large displacement $(\Delta y=15.5 \mu \mathrm{m})$. The colors indicate different displacement velocities as defined in Figure 2. (Top-left) Measurements with circular DNA. (Top-right) Measurements with linear DNA. ${ }^{12}$ (Bottom-left) Confining potential for circular DNA. (Bottom-right) Confining potential for linear DNA.

the linear DNA for displacements up to $\sim 1.5 \mu \mathrm{m}$, but the response with circular DNA deviated from linearity and was better described by a more slowly rising function of the form $F$ $=\alpha\left(1-\mathrm{e}^{-\beta \Delta y}\right)$, where $\alpha$ and $\beta$ are rate-dependent constants.

By integrating the force versus displacement data we can determine the potential per unit length $[U(y)]$ confining transverse displacements (Figure 2). We can then use the approach described by Zhou and Larson to determine the relative probability $[P(y)]$ that a chain segment thermally fluctuates a certain transverse distance $y$ from the primitive path via the relationship $P(y)=\mathrm{e}^{-U(y) / k T}$ (Figure 2). ${ }^{13} \mathrm{We}$ can then define a characteristic confining distance corresponding to the displacement where $U \cong 1 k T$ (i.e., $P(y) \cong 1 / \mathrm{e}$ ), as discussed previously. ${ }^{12}$ For the three fastest displacement rates, the confining distance was nearly the same for both topologies. However, for $0.5 \mu \mathrm{m} / \mathrm{s}$, the characteristic confining distance was twice as large for the circular DNA, and at the slowest rate $(0.1 \mu \mathrm{m} / \mathrm{s})$ there was negligible confinement with circular DNA, in contrast to the case with linear DNA. Thus, we have shown that tube confinements are less persistent on long time scales with circular DNA than with linear DNA.

As discussed previously, ${ }^{12}$ when comparing our results with the predicted tube radius in the $\mathrm{D}-\mathrm{E}$ model, one should probe the tube on a time scale close to the predicted thermal equilibration time $\tau_{\mathrm{e}}=a^{4} /\left(24 D_{\mathrm{G}} R_{\mathrm{G}}{ }^{2}\right){ }^{2}$ Using the value of $a$ predicted by $\mathrm{D}-\mathrm{E}$ theory, we calculate $\tau_{\mathrm{eL}} \cong 20 \mathrm{~ms}$ for linear DNA, and using the prediction of Iyer et al. $\left(a_{\mathrm{C}} \cong 0.7 a_{\mathrm{L}}\right)$, we calculate $\tau_{\mathrm{eC}} \cong 2.1 \mathrm{~ms}$ for circular DNA. Thus, in our previous measurements with linear DNA, we displaced the probe at $v_{\text {thL }}$ $=a_{\mathrm{L}} /\left(\tau_{\mathrm{eL}} \cong 20 \mathrm{~ms}\right) \cong 25 \mu \mathrm{m} / \mathrm{s}$, as well as testing lower and higher rates $(0.10-65 \mu \mathrm{m} / \mathrm{s})$. Here, we made measurements at the same rates with circular DNA so as to directly compare the two cases. However, according to the calculation above, the appropriate displacement rate for circular DNA for probing the tube on the thermal equilibration time would be different, $v_{\text {thC }}$ $=a_{\mathrm{C}} /\left(\tau_{\mathrm{eC}} \cong 2.1 \mathrm{~ms}\right) \cong 78 \mu \mathrm{m} / \mathrm{s}$. If we extrapolate our $13-65$ $\mu \mathrm{m} / \mathrm{s}$ force data to $78 \mu \mathrm{m} / \mathrm{s}$, we find $a_{\mathrm{C}} \cong 0.6 \mu \mathrm{m} \cong 0.75 a_{\mathrm{L}}$, which is very close to the prediction of Iyer et al. of $a_{\mathrm{C}} \cong 0.7 a_{\mathrm{L}}$.

While the small displacement data was similar for both topologies, in the circular case the force rose much more slowly with displacement for large displacements (Figure 3). The maximum force reached with circular DNA was $\sim 2-10$-fold

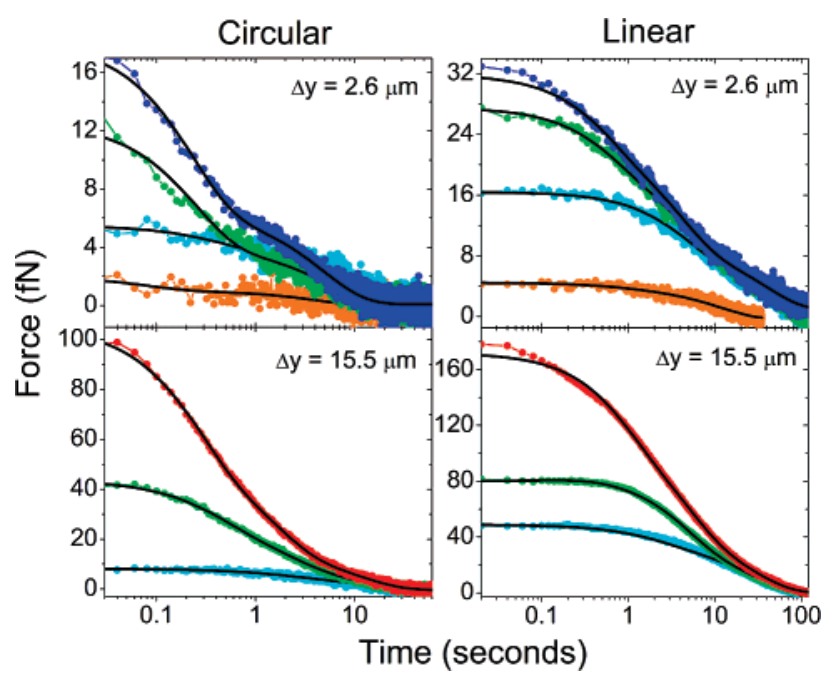

Figure 4. Measured force relaxation following the imposed transverse displacements of 2.6 or $15.5 \mu \mathrm{m}$. The colors indicate different displacement velocities as defined in Figure 2. The solid lines are fits to sums of two or three decaying exponentials. The linear DNA data is from our previous study. ${ }^{12}$

smaller than that with linear DNA, depending on the rate of displacement. A decrease in $\mathrm{d} F / \mathrm{d} y$ for $\Delta y>1 \mu \mathrm{m}$ was also apparent with circular DNA for all displacements rates (Figure 2 ). With linear DNA we observed a decrease in $\mathrm{d} F / \mathrm{d} y$ at a displacement of $\sim 5-10 \mu \mathrm{m}$, which is similar to the predicted primitive path length of the entangled linear DNA $\left(L_{0 \mathrm{~L}} \cong(N)\right.$ $\left.\left.N_{\mathrm{eL}}\right) a_{\mathrm{L}} \cong 9 \mu \mathrm{m}\right),{ }^{2}$ and we attributed this decrease to displacement-induced slippage of some of the entanglements off the probe $^{12}$ (Figure 3). Our present results with circular DNA are also consistent with this interpretation, because we see an earlier decrease in $\mathrm{d} F / \mathrm{d} y$ at $\sim 1-2 \mu \mathrm{m}$, and the predicted primitive path length for circular DNA is $L_{0 \mathrm{C}} \cong 2 \mu \mathrm{m}$. Such behavior observed in the large-displacement regime may be related to the phenomenon of convective constraint release, predicted by certain theories to be important in understanding the nonlinear response of polymeric liquids. ${ }^{35,41-44}$ With linear DNA we also observed a subsequent increase in $\mathrm{d} F / \mathrm{d} y$ for the $13 \mu \mathrm{m} / \mathrm{s}$ rate, which may be due to the probe encountering new entanglements. No such feature was observed with the circular DNA, again suggesting that entanglements tend to more easily slip off the probe as it is displaced. On the basis of our findings, we would expect convective constraint release to occur more readily with circular polymers than with linear polymers.

Further information came from the measured force relaxation following the imposed displacements (Figures 4 and 5). These measurements probe the relaxation of the distorted molecules surrounding the probe molecule. In our previous studies with linear DNA, we found three distinct exponential decay times, as mentioned in the Introduction. ${ }^{12}$ Here, with circular DNA we found that the relaxations following a small displacement $(\Delta y=2.6 \mu \mathrm{m})$ were well fit by the sum of just two decaying exponentials, with similar time constants for both large and small displacements and the different displacement rates. The relaxations following a large displacement $(\Delta y=15.5 \mu \mathrm{m})$ (with the exception of the slowest, $0.1 \mu \mathrm{m} / \mathrm{s}$ dataset) also revealed a third, longer relaxation time. To accurately determine the relaxation times, we fit the averaged data to a discrete sum of three decaying exponentials, finding $\tau_{1 \mathrm{C}}=0.3 \pm 0.1 \mathrm{~s}, \tau_{2 \mathrm{C}}=$ $4.1 \pm 1.6 \mathrm{~s}$, and $\tau_{3 \mathrm{C}}=11 \pm 2.1 \mathrm{~s}$. In comparison, with linear DNA we obtained $\tau_{1 \mathrm{~L}}=0.4 \pm 0.3 \mathrm{~s}, \tau_{2 \mathrm{~L}}=5.4 \pm 2.8 \mathrm{~s}$, and $\tau_{3 \mathrm{~L}}$ $=34 \pm 5.8 \mathrm{~s}$. Thus while $\tau_{1}$ and $\tau_{2}$ are similar for both 


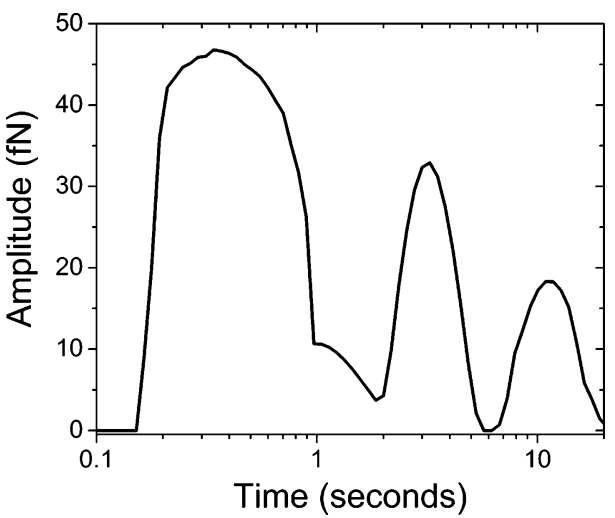

Figure 5. Spectrum of decay times determined by inverse Laplace transform of a typical force relaxation measurement following a transverse displacement $\Delta y=15.5 \mu \mathrm{m}$ at $65 \mu \mathrm{m} / \mathrm{s}$. The three peaks are centered at $\tau_{1} \cong 0.3 \mathrm{~s}, \tau_{2} \cong 3.3 \mathrm{~s}$, and $\tau_{3} \cong 12 \mathrm{~s}$.

topological cases, $\tau_{3}$ is much shorter for the circular molecules, and this mode was only observed with large displacements.

According to $\mathrm{D}-\mathrm{E}$ theory, the shortest relaxation time for an entangled polymer is the Rouse time ${ }^{2} \tau_{\mathrm{R}}=6 R_{\mathrm{G}}{ }^{2} / 3 \pi^{2} D_{\mathrm{G}}$, which is the time scale on which a deformed polymer elastically relaxes back to $L_{0}$. Using the values of $D_{\mathrm{G}}$ and $R_{\mathrm{G}}$ determined previously, ${ }^{34-36}$ we calculate $\tau_{\mathrm{RC}} \cong 0.14 \mathrm{~s}$ and $\tau_{\mathrm{RL}} \cong 0.6 \mathrm{~s}$, for the circular and linear cases, respectively. These values are close to the measured values. The longest predicted relaxation time in $\mathrm{D}-\mathrm{E}$ theory is the disengagement time $\tau_{\mathrm{D}}=\left(18 R_{\mathrm{G}}{ }^{2} / a^{2}\right) \tau_{\mathrm{R}}$, on which a polymer is predicted to reptate out of its deformed tube. For linear DNA, we calculate $\tau_{\mathrm{DL}} \cong 40 \mathrm{~s}$, in agreement with our measured value of $\tau_{3 \mathrm{~L}}=34 \pm 5.8 \mathrm{~s}$. In the case of circular DNA, Iyer et al. ${ }^{26}$ predict $\tau_{\mathrm{DC}} / \tau_{\mathrm{DL}}=\left(a_{\mathrm{C}}{ }^{2} / a_{\mathrm{L}}{ }^{2}\right) N^{2 v-1} \cong$ 0.2 , implying $\tau_{\mathrm{DC}} \cong 8 \mathrm{~s}$. Because the mechanism of reptation is usually discussed in the context of linear polymers, we emphasize here, as mentioned in the Introduction, that reptation of a circular polymer in the PPR framework refers to the reptation of the tree trunk structure, slowed by the frictional constraints of the protruding loops. Because the length of the trunk $\left(\sim N^{2 / 3}\right)$ is much shorter than a linear polymer of length $N$, it follows that the time for a circular polymer to reptate out of its tube is predicted to be much shorter than that for the linear case.

Following a $2.6 \mu \mathrm{m}$ displacement $(\sim 4$ times the characteristic confining length), we observed two relaxation times. The first $(0.3 \mathrm{~s})$ is consistent with Rouse relaxation and the second relaxation ( $4 \mathrm{~s}$ ) is an order of magnitude longer than the Rouse time. The observation of a transverse confining field that persists on a time scale much longer than the Rouse time is qualitatively consistent with reptation theory, which predicts that tube-like confinement would lead to two distinct relaxation time scales. However, the agreement with reptation theory is not quantitative, since Iyer et al. predict a disengagement time of $8 \mathrm{~s}$, which is a factor of 2 longer than the relaxation time we measure. The reason for this difference is unclear; however, a model proposed by Mhetar and Archer in modeling the response of entangled polymeric fluids to nonlinear step strains may provide one possible explanation. ${ }^{14}$ This model predicts that stress can be partially relaxed by reptation occurring on an intermediate time scale. The tube diameter is proposed to shrink during displacement as its length is increased, prohibiting complete relaxation of chain extension to $L_{0}$ in time $\tau_{\mathrm{R}}$. An additional relaxation mode with decay time between $\tau_{\mathrm{R}}$ and $\tau_{\mathrm{D}}$ is associated with the relaxation of the residual stretch as the tube expands back to its equilibrium diameter. Reptation out of the deformed tube occurs on this time scale as well; however, complete disengage- ment from the initial tube is still proposed to occur only after a time $\tau_{\mathrm{D}}$. As we discussed previously, ${ }^{12}$ evidence for a similar relaxation time has also been reported in nonlinear step shear experiments with linear polystyrene solutions ${ }^{45,46}$ and in flow deformation studies of linear DNA. ${ }^{35}$ While the nature of the deformation of the entangled polymers was different in the previous flow experiments than in our experiment, it is possible that this model may still be applied to the present case, since in both cases entangled polymers are deformed and allowed to relax.

The longer relaxation time scale of $\sim 11$ s observed following the larger displacement is in closer agreement with the predicted disengagement time of $8 \mathrm{~s}$. The measured ratio $\tau_{3 \mathrm{C}} / \tau_{3 \mathrm{~L}} \cong 0.3$ for circular vs linear polymers is also close to the predicted ratio of 0.2 for the disengagement times. It is not clear why this longer relaxation time only occurs following larger displacements, but within the context of the Mhetar-Archer model, we can speculate that reptation occurring on an intermediate time scale may be sufficient for circular polymers to completely disengage from their deformed tubes following small (but not large) deformations. That a relaxation time corresponding to the disengagement time occurs with linear DNA for small displacements may be connected with the finding of larger confining forces for linear DNA ( $\geq 2$ times larger than with circular DNA at $2.6 \mu \mathrm{m}$ ). Perhaps a weaker, less persistent confining field leads to less deformation, and reduces the time needed for the polymer to reptate into an equilibrium tube configuration.

At long times approaching $\tau_{\mathrm{D}}$, we expect the tube-like constraints to weaken and eventually disappear. Indeed, at the lowest displacement rate $(0.1 \mu \mathrm{m} / \mathrm{s})$, we found negligible forces resisting transverse displacement of the probe molecule. That this displacement rate corresponds to $\sim 10 a_{\mathrm{L}} / \tau_{\mathrm{DL}}$ for the linear case but only $\sim 2 a_{\mathrm{C}} / \tau_{\mathrm{DC}}$ for the circular case is consistent with our finding of a much lower confining force with circular DNA at this lowest displacement rate.

Acknowledgment. We thank Peter Rickgauer, Al Schweizer, and Derek Fuller for technical assistance and Ron Larson, Ashish Lele, and Balaji Iyer for many helpful discussions. We acknowledge funding from the Petroleum Research Fund of the American Chemical Society, a fellowship from the Sloan Foundation (to D.E.S.), and a graduate fellowship from the NSF (to R.M.R.).

\section{References and Notes}

(1) de Gennes, P. G. Scaling Concepts in Polymer Physics; Cornell University Press: Ithaca, NY, 1979.

(2) Doi, M.; Edwards, S. F., The theory of polymer dynamics; Oxford University Press: Oxford, 1986.

(3) McLeish, T. C. B. Adv. Phys. 2002, 51 (6), 1379-1527.

(4) Roovers, J. Macromolecules 1985, 18 (6), 1359-1361.

(5) Roovers, J. Macromolecules 1988, 21 (5), 1517-1521.

(6) McKenna, G. B.; Hadziioannou, G.; Lutz, P.; Hild, G.; Strazielle, C.; Straupe, C.; Rempp, P.; Kovacs, A. J. Macromolecules 1987, 20 (3), 498-512.

(7) Orrah, D. J.; Semlyen, J. A.; Rossmurphy, S. B. Polymer 1988, 29 (8), 1452-1454

(8) Cosgrove, T.; Griffiths, P. C.; Hollingshurst, J.; Richards, R. D. C.; Semlyen, J. A. Macromolecules 1992, 25 (25), 6761-6764.

(9) Cosgrove, T.; Turner, M. J.; Griffiths, P. C.; Hollingshurst, J.; Shenton, M. J.; Semlyen, J. A. Polymer 1996, 37 (9), 1535-1540.

(10) von Meerwall, E.; Ozisik, R.; Mattice, W. L.; Pfister, P. M. J. Chem. Phys. 2003, 118 (8), 3867-3873.

(11) Robertson, R. M.; Smith, D. E. Macromolecules 2007, 40 (9), 33733377. Robertson, R. M.; Smith, D. E. PNAS 2007, 104 (12), 48244827.

(12) Robertson, R. M.; Smith, D. E. Phys. Rev. Lett. 2007, 99 (12), 126001.

(13) Zhou, Q.; Larson, R. G. Macromolecules 2006, 39 (19), 6737-6743. 
(14) Mhetar, V.; Archer, L. A. J. Non-Newtonian Fluid Mech. 1999, 81 $(1-2), 71-81$

(15) Klein, J. Macromolecules 1986, 19 (1), 105-118.

(16) Cates, M. E.; Deutsch, J. M. J. Phys. 1986, 47 (12), 2121-2128.

(17) Obukhov, S. P.; Rubinstein, M.; Duke, T. Phys. Rev. Lett. 1994, 73 (9), 1263-1266.

(18) McLeish, T. Science 2002, 297 (5589), 2005-2006.

(19) Muller, M.; Wittmer, J. P.; Cates, M. E. Phys. Rev. E 1996, 53 (5), 5063-5074.

(20) Brown, S.; Szamel, G. J. Chem. Phys. 1998, 109 (14), 6184-6192.

(21) Muller, M.; Wittmer, J. P.; Cates, M. E. Phys. Rev. E 2000, 61 (4), 4078-4089.

(22) Ozisik, R.; Doruker, P.; Mattice, W. L.; von Meerwall, E. D. Comput. Theor. Polym. Sci. 2000, 10 (5), 411-418.

(23) Ozisik, R.; von Meerwall, E. D.; Mattice, W. L. Polymer 2002, 43 (2), 629-635.

(24) McLeish, T. C. B.; Larson, R. G. J. Rheol. 1998, 42 (1), 81-110.

(25) Karayiannis, N. C.; Mavrantzas, V. G. Macromolecules 2005, 38 (20), $8583-8596$

(26) Iyer, B. V. S.; Lele, A. K.; Juvekar, V. A. Phys. Rev. E 2006, 74 (2), 021805.

(27) Colby, R. H.; Rubinstein, M. Macromolecules 1990, 23 (10), 27532757.

(28) Heo, Y.; Larson, R. G. J. Rheol. 2005, 49 (5), 1117-1128.

(29) Putz, M.; Kremer, K.; Grest, G. S. Europhys. Lett. 2000, 49 (6), 735741.

(30) Everaers, R.; Sukumaran, S. K.; Grest, G. S.; Svaneborg, C.; Sivasubramanian, A.; Kremer, K. Science 2004, 303 (5659), 823826.

(31) Kremer, K.; Sukumaran, S. K.; Everaers, R.; Grest, G. S. Comput. Phys. Commun. 2005, 169 (1-3), 75-81.

(32) Sukumaran, S. K.; Grest, G. S.; Kremer, K.; Everaers, R. J. Polym. Sci. Part B-Polym. Phys. 2005, 43 (8), 917-933.
(33) Sen, S.; Kumar, S. K.; Keblinski, P. Macromolecules 2005, 38 (3), $650-653$.

(34) Robertson, R. M.; Laib, S.; Smith, D. E. Proc. Natl. Acad. Sci. U.S.A. 2006, 103 (19), 7310-7314.

(35) Teixeira, R. E.; Dambal, A. K.; Richter, D. H.; Shaqfeh, E. S. G.; Chu, S. Macromolecules 2007, 40 (7), 2461-2476.

(36) Hur, J. S.; Shaqfeh, E. S. G.; Babcock, H. P.; Smith, D. E.; Chu, S. J. Rheol. 2001, 45 (2), 421-450.

(37) Jary, D.; Sikorav, J. L.; Lairez, D. Europhys. Lett. 1999, 46 (2), 251255.

(38) Fuller, D. N.; Gemmen, G. J.; Rickgauer, J. P.; Dupont, A.; Millin, R.; Recouvreux, P.; Smith, D. E. Nucleic Acids Res. 2006, 34 (2), e15.

(39) Laib, S.; Robertson, R. M.; Smith, D. E. Macromolecules 2006, 39 (12), 4115-4119.

(40) Rickgauer, J. P.; Fuller, D. N.; Smith, D. E. Biophys. J. 2006, 91 (11), 4253-4257.

(41) Marrucci, G. J. Non-Newtonian Fluid Mech. 1996, 62 (2-3), 279289.

(42) Mead, D. W.; Larson, R. G.; Doi, M. Macromolecules 1998, 31 (22), 7895-7914.

(43) Ianniruberto, G.; Marrucci, G. J. Non-Newtonian Fluid Mech. 1996, 65 (2-3), 241-246.

(44) Muller, R.; Pesce, J. J.; Picot, C. Macromolecules 1993, 26 (16), 43564362.

(45) Archer, L. A.; Sanchez-Reyes, J.; Juliani Macromolecules 2002, 35 (27), 10216-10224.

(46) Sanchez-Reyes, J.; Archer, L. A. Macromolecules 2002, 35 (13), 5194-5202.

MA071440E 